\title{
PN G291.4-00.3: A new type I planetary nebula*
}

\author{
D. Nürnberger ${ }^{1,2}$, S. Durand ${ }^{3,4}$, J. Köppen ${ }^{4,5,6}$, Th. Stanke ${ }^{7}$, M. Sterzik ${ }^{8}$, and S. Els ${ }^{8,9}$ \\ 1 Institut für Theoret. Physik und Astrophysik, Univ. Würzburg, Am Hubland, 97074 Würzburg, Germany \\ 2 Institut de Radio-Astronomie Millimétrique, 300 rue de la Piscine DU, 38406 St. Martin-d'Hères, France \\ 3 Inst. Astronômico e Geofísico, Univ. de São Paulo, Av. Miguel Stéfano 4200, 04301 - 904 São Paulo SP, Brazil \\ 4 Observatoire Astronomique de Strasbourg, 11 rue de l'Université, 67000 Strasbourg, France \\ 5 Institut für Theoretische Physik und Astrophysik, Universität Kiel, Leibnizstraße 15, 24098 Kiel, Germany \\ 6 International Space University, Parc d'Innovation, 67400 Illkirch, France \\ 7 Max-Planck-Institut für Radioastronomie, Auf dem Hügel 69, 53121 Bonn, Germany \\ 8 European Southern Observatory, Casilla 19001, Santiago 19, Chile \\ 9 Institut für Theoretische Astrophysik, Univ. Heidelberg, Tiergartenstraße 15, 69121 Heidelberg, Germany
}

Received 4 August 2000 / Accepted 27 July 2001

\begin{abstract}
In the vicinity of the southern hemisphere giant H II region NGC 3603 we discovered a new planetary nebula: PN G291.4-00.3 located at RA $\mathrm{J}_{2000.0}=11^{\mathrm{h}} 14^{\mathrm{m}} 32 .{ }^{\mathrm{s}} 1 \pm 0 . \mathrm{s} 3, \mathrm{DEC}_{\mathrm{J} 2000.0}=-61^{\circ} 00^{\prime} 02^{\prime \prime} \pm 1^{\prime \prime}$. Monochromatic images reveal a central ring-like structure accompanied by onsets of arc-like filaments which might outline a bipolar outflow. Analysis of the nebula spectrum shows that helium and nitrogen are enriched, while oxygen, sulphur and argon abundances seem to be solar. The central star must be hot (above $100 \mathrm{kK}$ ) and having a luminosity of more than $3000 L_{\odot}$. All evidence shows that PN G291.4-00.3 is a high-excitation, density bounded planetary nebula of the type I, coming from the high-mass end of the intermediate mass stars.
\end{abstract}

Key words. ISM: individual objects: NGC 3603 - planetary nebulae: general - planetary nebulae: individual: PN G291.4-00.3

\section{Introduction}

Planetary nebulae $(\mathrm{PNe})$ represent the penultimate state in the evolution of intermediate mass stars. They consist of a shell of gas ionized and excited by the ultraviolet radiation of the very hot central star before it fades into a white dwarf. The nebula's shell contains material ejected by the star and being returned to the interstellar medium.

Among them, there is a class of objects with bipolar and/or filamentary morphology. Most of them are type I objects $(\mathrm{He} / \mathrm{H} \geq 0.125$ and $\log (\mathrm{N} / \mathrm{O}) \geq-0.3$; Peimbert \& Torres-Peimbert 1987). They have large expansion velocities and are mostly found in the galactic plane, with a scale-height of $130 \mathrm{pc}$. Their central stars lie in the HR diagram generally on the tracks of higher masses (Corradi \& Schwarz 1995). Thus, their progenitor stars must have been rather massive and one may expect that helium and nitrogen synthesized in the star are ejected via the nebula. However, theoretical calculations do not yet permit to reproduce the observed abundances in a quantitative way.

Send offprint requests to: D. Nürnberger, e-mail: nurnberg@astro.uni-wuerzburg.de

* Based on observations obtained at the European Southern Observatory, La Silla, Chile.
In this paper we report the discovery of a new type I PN in the Galaxy: Sect. 2 deals with the detection on a wide field image, Sect. 3 discusses the follow-up narrow band imaging observations and Sect. 4 presents the analysis of spectral data. In Sect. 5 we discuss the evolutionary status of the nebula and the central star.

\section{Detection: WFI imaging}

PN G291.4-00.3 was discovered serendipitously on wide field CCD exposures centered on the giant HII region NGC 3603. These images were obtained with the Wide Field Imager (WFI) mounted at the ESO/MPG $2.2 \mathrm{~m}$ telescope on La Silla on Jan. 21st 1999 under moderate seeing conditions $\left(\sim 1^{\prime \prime}\right)$.

The camera used a mosaic of 8 CCDs, each a $2 \mathrm{k} \times 4 \mathrm{k}$ pixel array, providing a total detector size of $8 \mathrm{k} \times 8 \mathrm{k}$ pixels with a field-of-view of about $0.54 \times 0.54$ at an image scale of 0.238 per pixel. Images were taken through narrow-band filters at $6580 \AA$ ( $\mathrm{H} \alpha \&[\mathrm{~N} \mathrm{II}])$ and $6760 \AA$ ([S II]), as well as a medium-passband filter at $8160 \AA$ which was used to measure continuum emission. Integration times for each filter were 10 minutes, split into 5 dithered exposures to compensate for the gaps between the CCDs, bad pixels and cosmics. Some basic information 

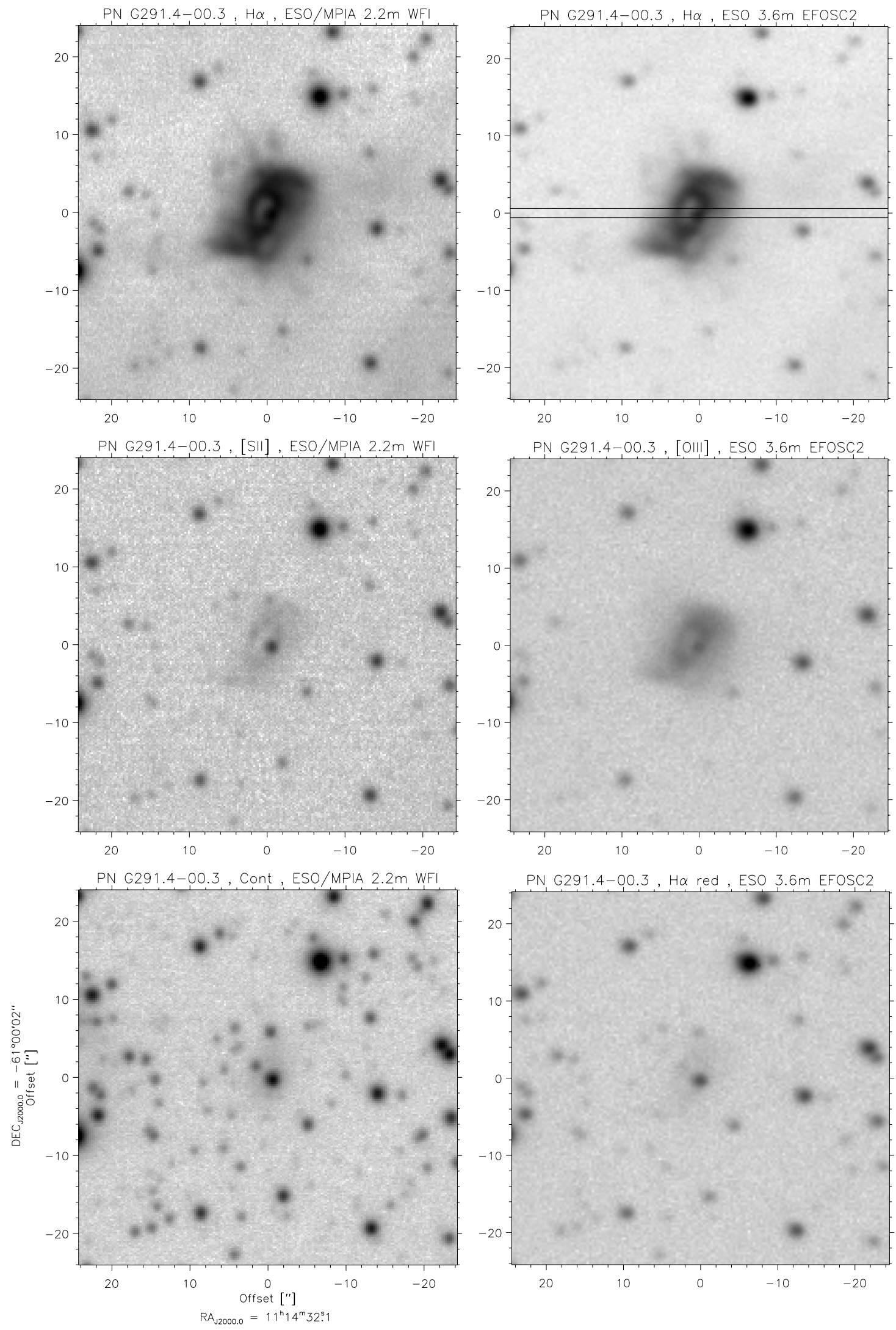

Fig. 1. Imaging results obtained with narrow band filters centered on the $\mathrm{H} \alpha$, [O III] and [S II] lines as well as line free continuum (8160 $\AA$ and $6659 \AA$, respectively). The data sets were taken with the WFI mounted at the ESO / MPG $2.2 \mathrm{~m}$ telescope (left panels) and with EFOSC 2 at the ESO $3.6 \mathrm{~m}$ telescope (right panels). Intensities are scaled logarithmic. Position and orientation of the $1^{\prime \prime} 2$ wide slit used for spectroscopy is given in the upper right panel. 
Table 1. Overview on the obtained data sets.

\begin{tabular}{|c|c|c|c|c|c|c|c|}
\hline \multicolumn{8}{|c|}{ Imaging } \\
\hline telescope & date & $\begin{array}{c}\text { UT } \\
\text { (start) }\end{array}$ & $\begin{array}{c}\text { airmass } \\
\text { (start-end) }\end{array}$ & filter & $\begin{array}{l}\lambda_{\mathrm{c}} \\
{[\AA]}\end{array}$ & $\begin{array}{l}\Delta \lambda \\
{[\AA]}\end{array}$ & $\begin{array}{c}\text { exp. time } \\
{[\mathrm{s}]}\end{array}$ \\
\hline $\mathrm{ESO} / \mathrm{MPG} 2.2 \mathrm{~m} \& \mathrm{WFI}$ & 21.01 .99 & $08: 30: 21$ & $1.19-1.19$ & $\# 856 \mathrm{H} \alpha \&[\mathrm{~N} \mathrm{II}]$ & 6585 & 70 & 600 \\
\hline & & $08: 48: 20$ & $1.20-1.20$ & \# 857 [S II] & 6760 & 50 & 600 \\
\hline & & 08:12:07 & $1.18-1.18$ & \# 851 Cont & 8160 & 240 & 600 \\
\hline ESO $3.6 \mathrm{~m} \&$ EFOSC 2 & 08.08 .99 & $23: 12: 34$ & $1.66-1.68$ & \# $694 \mathrm{H} \alpha \&[\mathrm{~N} \mathrm{II}]$ & 6577 & 62 & 300 \\
\hline & & $23: 24: 00$ & $1.72-1.74$ & \# 689 [O III] & 5004 & 56 & 300 \\
\hline & & $23: 18: 16$ & $1.69-1.71$ & \#698 H $\alpha$ red & 6659 & 61 & 300 \\
\hline \multicolumn{8}{|c|}{ Spectroscopy } \\
\hline telescope & date & $\begin{array}{c}\text { UT } \\
\text { (start) }\end{array}$ & $\begin{array}{c}\text { airmass } \\
\text { (start-end) }\end{array}$ & grism & $\begin{array}{c}\lambda \text { range } \\
{[\AA]}\end{array}$ & $\begin{array}{c}\text { disp. } \\
{[\AA / \text { pix }]}\end{array}$ & $\begin{array}{c}\text { exp. time } \\
{[\mathrm{s}]}\end{array}$ \\
\hline ESO $3.6 \mathrm{~m} \&$ EFOSC 2 & 07.08 .99 & $23: 38: 26$ & $1.77-1.83^{[\mathrm{a}]}$ & $\# 11$ & $3215-7319$ & 4 & 600 \\
\hline & & $23: 26: 56$ & $1.71-1.76^{[\mathrm{b}]}$ & \# 12 & $6025-10286$ & 4 & 600 \\
\hline
\end{tabular}

Notes: $[\mathrm{a}]$ rotator angle $=259^{\circ} 6$, parallactic angle $=89^{\circ} .4 \ldots 99^{\circ} .6$; $[\mathrm{b}]$ rotator angle $=259.6$, parallactic angle $=86^{\circ} .9 .89^{\circ} 1$.

on the used filters is given in Table 1 . The faintest sources detected in the continuum filter have $I$ band magnitudes of the order $22 \mathrm{mag}$.

The images were reduced using standard IRAF procedures. While photometric calibration was applied on the $\mathrm{H} \alpha$ image, astrometric calibration was performed on the WFI continuum image by making use of the Digitized Sky Survey 2 (DSS2) and the TYCHO-2 catalogue (Hog et al. 2000), both accessible e.g. via the Centre de Données astronomiques de Strasbourg (CDS). In a first step, nearby TYCHO-2 sources were registered on the DSS2 image and afterwards identified on the WFI continuum mosaic: 8959-317-1, 8959-478-1, 8959-2415-1, 8959-2476-1 and $8959-2563-1$. Note that the most nearby TYCHO-2 source 8959-2437-1, which is the brightest source of the finding chart presented in Fig. 2, is unfortunately located just outside the field-of-view of our WFI data.

Then, within the ICRS reference frame we determined the position of the continuum source visible in the western part of the ring-like PN feature (Fig. 1; for a discussion of its possible association with the PN see Sect. 3). Its position is $\mathrm{RA}_{\mathrm{J} 2000.0}=11^{\mathrm{h}} 14^{\mathrm{m}} 31 . \mathrm{s} 9, \mathrm{DEC}_{\mathrm{J} 2000.0}=$ $-61^{\circ} 00^{\prime} 02^{\prime \prime} .3$ with a nominal accuracy of about \pm 0. .'3 in both right ascension and declination. This value appears reasonable as it is of the order of the WFI pixel scale $\left(00^{\prime \prime} 238\right)$. Comparison with the USNO-A2.0 catalogue yields $0225-10792553\left(\mathrm{RA}_{\mathrm{J} 2000.0}=11^{\mathrm{h}} 14^{\mathrm{m}} 32.01\right.$, $\mathrm{DEC}_{\mathrm{J} 2000.0}=-61^{\circ} 00^{\prime} 02^{\prime \prime} .1 ; R=11^{\mathrm{m}} .7$ and $B=16^{\mathrm{m}} 3$ ) to be a likely counterpart of our continuum source.

Anyway, as the center position of the $\mathrm{PN}$ itself is only poorly defined by the ring-like feature but appears to be offset from the continuum source by about $+1^{\prime \prime}$. 4 in right ascension and +0 ". 4 in declination (Fig. 1), the positional accuracy should be restricted to $\pm 2^{\prime \prime}$ in right ascension and $\pm 1^{\prime \prime}$ in declination. Taking into account this limitation, our best estimate for the $\mathrm{PN}$ position is
$\mathrm{RA}_{\mathrm{J} 2000.0}=11^{\mathrm{h}} 14^{\mathrm{m}} 32 . \mathrm{s} 1 \pm 0 . \mathrm{s} 3, \mathrm{DEC}_{\mathrm{J} 2000.0}=-61^{\circ} 00^{\prime} 02^{\prime \prime}$ $\pm 1^{\prime \prime}$.

By cross-checking the source position with several catalogues - e.g. the Strasbourg-ESO Catalogue of Galactic Planetary Nebulae (Acker et al. 1992), the IRAS Catalogues (Beichman et al. 1988) and a survey of $\mathrm{OH}$ maser sources (Caswell 1998) - as well as by searching in the CDS-SIMBAD astronomical database we realized that this object had not been properly identified and characterized before. Moreover, in a recent survey of the southern zone of avoidance it was misclassified as a galaxy (RKK 2906; RA $\mathrm{J}_{2000.0}=11^{\mathrm{h}} 14^{\mathrm{m}} 32 .{ }^{\mathrm{s}} 0$, $\mathrm{DEC}_{\mathrm{J} 2000.0}=-61^{\circ} 00^{\prime} 03^{\prime \prime}$; Kraan-Korteweg 2000).

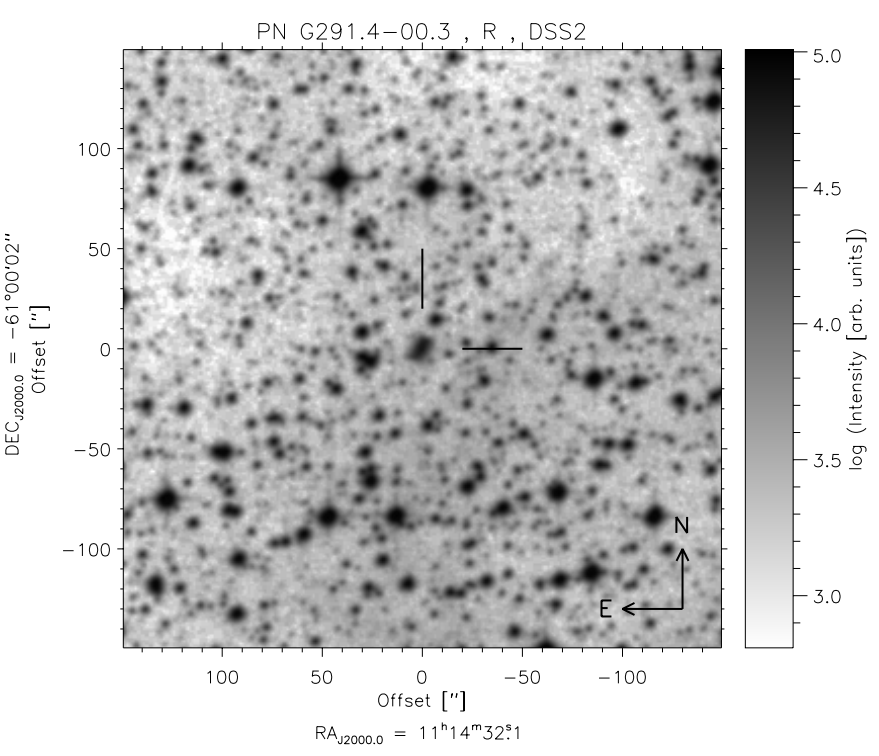

Fig. 2. Finding chart of PN G291.4-00.3 (DSS2 R plate). 


\section{Morphology: EFOSC 2 imaging}

Follow-up narrow band imaging observations were performed on Aug. 8th, 1999 at the ESO $3.6 \mathrm{~m}$ telescope (the finding chart of the $\mathrm{PN}$ is presented in Fig. 2). Details of the observations are summarized in Table 1. Figure 1 shows the images taken: similarly to the WFI data PN G291.4-00.3 appears as a bright nebulosity in the $\mathrm{H} \alpha \&[\mathrm{NII}]$ filter, is quite faint in [O III] and not detectable in the continuum (diameter $\sim 11^{\prime \prime}$ ).

Its overall appearance consists of a roughly northsouth elongated ring-like structure with short onsets of spiral-like arms at the northern and southern tips, which is superposed on rhomb-like shaped diffuse emission. The central ring appears to be tilted by $50^{\circ}$ to $55^{\circ}$ against the plane of sky. Its diameter is about $6^{\prime \prime}$ along the major axis. The arc-like shaped filaments extending the ring are suggestive of a wide-angled hourglass-shaped bipolar outflow $\left(\mathrm{PA} \sim 80^{\circ}\right)$.

In the light of $\mathrm{H} \alpha \&[\mathrm{NII}]$ we can distinguish roughly at the north and south side of the $\mathrm{PN}$ one or two very faint pair(s) of jet-like structures which are not visible in the [O III] or $[\mathrm{S} \mathrm{II}]$ images. A closer study of the orientation and symmetry of those bipolar structures might allow to localize the central star, because each pair is always oriented along opposite radial directions from the central star (Balick 1987).

On our WFI continuum image (Fig. 1; detection limit corresponding to $I \sim 22^{\mathrm{m}}$ ) no star is found at the very center of the nebula. But there are two stars near the center of the nebula which - at first sight - appear as possible candidates for or companions of the central star. A fairly bright one, located in the western part of the ring and likely to be USNO-A2.0 0225-10792553 $\left(R=11^{\mathrm{m}} \mathrm{7}\right.$, $B=16 .{ }^{\mathrm{m}} 3$; see Sect. 2 ), is visible in all six panels of Fig. 1 . Another, fainter star coincides with the eastern part of the ring.

Both are located about $1.5^{\prime \prime}$ from the PN center which corresponds to a projected distance of at least $9000 \mathrm{AU}$ or $0.04 \mathrm{pc}$ at a heliocentric distance of $6 \mathrm{kpc}$ (Sect. 5). The true separation therefore would be comparable to or larger than the largest values found for wide binary central stars of PNe (e.g. $10600 \mathrm{AU}$ is estimated for Abell 30 by Ciardullo et al. 1999), making a real physical association with our nebula rather unlikely.

It is more plausible that both sources are simply field stars: On the continuum image of Fig. 1 we count 15 stars which are at least as bright as the star in the western part of the ring. This suggests that one such star is expected to be found every $12^{\prime \prime} \times 12^{\prime \prime}$, which roughly corresponds to the area covered by the PN. For the fainter star a similar consideration holds.

\section{Nebular properties: Spectroscopy}

\subsection{Observational material}

Spectroscopic observations were performed using EFOSC 2 at the ESO $3.6 \mathrm{~m}$ telescope on Aug. 7th, 1999.

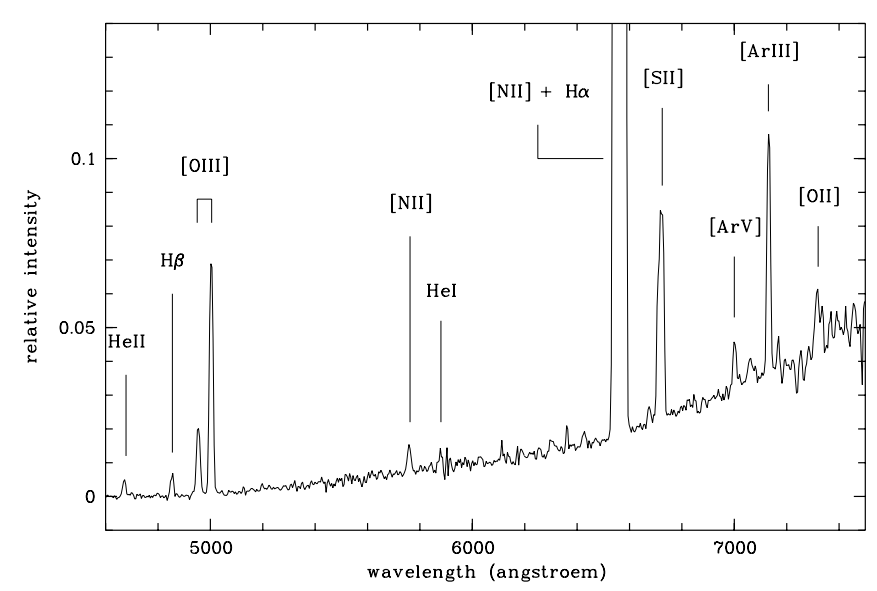

Fig. 3. Optical spectrum of PN G291.4-00.3 obtained with EFOSC 2 at the ESO $3.6 \mathrm{~m}$ telescope.

Table 2. Observed and dereddened line fluxes of PN G291.4-00.3, normalized to $F(\mathrm{H} \beta)=100$. The fluxes marked with an asterisk are scaled from Cuisinier's spectrum (see text). The wavelength calibration is limited by the spectral resolution $(4 \AA)$ of the spectrum. The errors of the flux calibration are typically $10 \%$ for the strongest lines and up to a factor of 2 for the weakest lines. Contributions to the error budget are discussed in the text.

\begin{tabular}{|r|l|r|r|}
\hline$\lambda[\AA]$ & ident. & observed & dereddened \\
\hline \hline 4686 & He II & 89 & 113 \\
4861 & $\mathrm{H} \beta$ & 100 & 100 \\
4959 & {$[\mathrm{O} \mathrm{III}]$} & 328 & 270 \\
5007 & {$[\mathrm{O} \mathrm{III}]$} & 1061 & 792 \\
5754 & {$[\mathrm{~N} \mathrm{II}]$} & 153 & 21 \\
5876 & $\mathrm{HeI}$ & $87:$ & $10:$ \\
6548 & {$[\mathrm{~N} \mathrm{II}]$} & $4400^{*}$ & 256 \\
6563 & $\mathrm{H} \alpha$ & $5000^{*}$ & 283 \\
6583 & {$[\mathrm{~N} \mathrm{II}]$} & $13700^{*}$ & 758 \\
6678 & $\mathrm{He} \mathrm{I}$ & $90:$ & $4:$ \\
6716 & {$[\mathrm{~S} \mathrm{II}]$} & 756 & 35 \\
6731 & {$[\mathrm{~S} \mathrm{II}]$} & 933 & 43 \\
7006 & {$[\mathrm{Ar} \mathrm{V}]$} & 224 & 6 \\
7136 & {$[\mathrm{Ar} \mathrm{III}]$} & 1061 & 27 \\
7325 & {$[\mathrm{O} \mathrm{II}]$} & 394 & 9 \\
\hline
\end{tabular}

We used a 1 .' 2 wide slit on grisms \#11 and \#12 covering the wavelength range from about 3200 to $10300 \AA$ at a dispersion of $4 \AA$ per pixel. Position and orientation of the slit are indicated in Fig. 1, corresponding rotator and parallactic angles are given in Table 1. The slit was always kept oriented perpendicular to the horizon, to minimize the influence of differential refraction. The total exposure time was $10 \mathrm{~min}$ for each part of the spectrum and the seeing was measured to be about 0. . 6 .

The data was reduced in the usual way. However, for technical reasons no arc spectra could be taken for 
grism \#11. We therefore used in the blue part the nebular lines for a rough wavelength calibration. Furthermore, no observations of standard stars could be done. We corrected for the overall wavelength response of the system by using the measured throughput provided by the staff of the $3.6 \mathrm{~m}$ telescope ${ }^{1}$. The red and blue spectra were scaled in flux as to give in the region of their overlap (6050 to $7300 \AA$ ) a good match in both the continuum and the six line features in common (better than 10 percent in the stronger lines).

The CCD image of the red spectrum shows rather strong ripples. As the detector's quantum efficiency drops strongly longward of $7500 \AA$, corrections become rather uncertain at these wavelengths. Therefore, we decided to exclude the reddest part $(\lambda>7500 \AA)$ when combining the blue and red spectrum. But nevertheless it should be mentioned that the [S III] lines at $9069 \AA$ and $9532 \AA$ are quite strong, their flux being about half of that of $\mathrm{H} \alpha+[\mathrm{N} \mathrm{II}]$.

In the range from $4000 \AA$ to $7500 \AA$ (Fig. 3 ) the spectrum exhibits emission lines typical of a highly ionized planetary nebula, such as He II 4686 and [O III] 5007, as well as lines from low ionization species such as [N II] and [S II]. The continuum rising towards the red is due to the star USNO-A2.0 0225-10792553, which is superposed on the western part of the ring as mentioned above. Its red colour thus makes it even less likely to be the central star of the nebula.

The $\mathrm{H} \alpha$ line is completely blended with the [N II] 6583 and 6548 lines. The [S II] doublet at $6731 \AA$ and $6717 \AA$ is marginally resolved and is treated by multi-gaussian fitting. The observed line fluxes are presented in Table 2. If there were no calibration problems with the data, the measurement errors on the line fluxes - relative to $\mathrm{H} \beta$ - would be estimated to be about $10 \%$ for the strongest lines, going up to a factor of two for the weakest lines. From a comparison of the throughputs measured with different stars and at different times, we estimate that the uncertainties of the wavelength dependent response are of the order of $20 \%$.

Recently, F. Cuisinier (priv. comm.) took highresolution $(0.25 \AA)$ long-slit spectra of the $\mathrm{PN}$ near $\mathrm{H} \alpha$ with the $1.6 \mathrm{~m}$ telescope at the Pico dos Dias Observatory (Brazil). Based on this data set the $\mathrm{H} \alpha$ flux from the entire nebula is determined as $[3.8 \pm 0.4] \times 10^{-13} \mathrm{erg} \mathrm{cm}^{-2} \mathrm{~s}^{-1}$. The dereddened $[\mathrm{N} \mathrm{II}] / \mathrm{H} \alpha$ ratio is found to be $2.6 \pm 0.3$. We use these more reliable data in place of our own, by scaling our data to give the same $\mathrm{H} \alpha+[\mathrm{NII}]$ flux. This is legitimate because our low resolution spectrum is not affected by differential atmospheric refraction (see Sect. 4.1).

The high-resolution spectra show two components of the $[\mathrm{N} \mathrm{II}]$ lines: the NE part of the ring has a radial (heliocentric) velocity of $-6.5 \mathrm{~km} \mathrm{~s}^{-1}$, while $+15 \mathrm{~km} \mathrm{~s}^{-1}$ is measured for the SW part, with an accuracy estimated to be $\pm 1.5 \mathrm{~km} \mathrm{~s}^{-1}$ (for $90 \%$ confidence). The velocities at other positions conform with the interpretation of a bipolar

\footnotetext{
1 http://www.ls.eso.org/lasilla/Telescopes/360cat/
}

Table 3. Results of the plasma diagnostics of PN G291.4-00.3. The third column gives the abundances obtained with adopted electron temperature and density.

\begin{tabular}{|c|r|r|}
\hline$c$ & $2.8 \pm 0.2$ & - \\
$E C$ & 7 & - \\
$n_{\mathrm{e}}\left[\mathrm{cm}^{-3}\right]$ & $1200_{-600}^{+1200}$ & 1200 \\
$T_{\mathrm{e}}[\mathrm{K}]$ & $12700_{-1400}^{+1500}$ & 10000 \\
\hline \hline $\mathrm{He}$ & 11.23 & 11.23 \\
$\mathrm{~N}$ & 9.00 & 9.04 \\
$\mathrm{O}$ & 8.58 & 8.94 \\
$\mathrm{~S}$ & 7.41 & 7.31 \\
$\mathrm{Ar}$ & 6.34 & 6.58 \\
\hline
\end{tabular}

flow with a deprojected velocity of about $20 \mathrm{~km} \mathrm{~s}^{-1}$ and the SW part of the ring and its associated arms receeding. The radial velocity of the $\mathrm{PN}$ is $+5 \mathrm{~km} \mathrm{~s}^{-1}$.

\subsection{Plasma diagnostics}

We use the plasma diagnostics code HOPPLA (for a description see Acker et al. 1989; Köppen et al. 1991) to derive the basic plasma parameters from the spectrum:

- The observed intensity ratio of the $\mathrm{H} \alpha$ and $\mathrm{H} \beta$ lines of $49 \pm 8$ gives an extinction constant $c=3.8 \pm 0.2$;

- This gives a $\mathrm{H} \beta$ flux of $\log F(\mathrm{H} \beta)=-14.1$, just below the detection limit for the nebulae in the catalogue of Acker et al. (1992), which is about -14.0 (Köppen, unpublished);

- From the ratio of the [S II] 6731 and 6717 lines we derive an electron density of $n_{\mathrm{e}}=1200_{-600}^{+1200} \mathrm{~cm}^{-3}$;

- The electron temperature $T_{\mathrm{e}}=12700_{-1400}^{+1500} \mathrm{~K}$ in the $\mathrm{N}^{+}$ zone is obtained from the dereddened ratio of the [N II] lines at $6583+6548$ and $5754 \AA$.

The program performs the above analysis iteratively until all three quantities have sufficiently converged.

Since He II 4686 is as strong as $\mathrm{H} \beta$, an excitation class of 7 can be assigned. However, the [O III] lines are weaker than one would expect for such high excitation, being only about 10 times stronger than $\mathrm{H} \beta$. A most likely explanation is that the nebula is density bounded, with the nebula's outer edge being right inside the $\mathrm{O}^{++}$zone. For a spherically symmetric object this would imply that no emission from low ionization species should be observable. The clear presence of such emission indicates that the nebula is strongly matter bounded in some directions, but nearly ionization bounded in others. This conforms neatly with the bipolar appearence on the images.

With the derived electron temperature and density we finally determine the ionic and - with standard ionization correction factors - the elemental abundances. The He I lines at $5876 \AA$ and $6678 \AA$ have an intensity ratio of 2.5 , much smaller than the theoretical ratio of 3.5. Evidently, the $6678 \AA$ line is close to the noise level and has been overestimated. The value taken from the $5876 \AA$ line is accepted. 
The results of the plasma diagnostics are collected in Table 3. As the obtained electron temperature is rather high in comparison with other type I nebulae (see the next section), we also show the results derived with a reduced temperature. We consider these values more reliable. In both cases the enhanced values of helium and nitrogen abundances clearly identify our object as a genuine type I planetary nebula.

Gruenwald \& Viegas (1998) have checked the application of the plasma diagnostics method with spherical and bipolar photoionization models. The helium abundance and the abundance ratio $\mathrm{N} / \mathrm{O}$ tend to be progressivly overestimated in nebulae around hot stars. They show that for stellar temperatures between 100 and $200 \mathrm{kK}$, this error is less than 0.05 dex for $\mathrm{He} / \mathrm{H}$ and less than 0.3 dex for N/O. We tried to check directly our plasma diagnostics with calculated spectra of 3-D models, although the sets of lines available were different from the one we used for our PN: The moderately excitation spectrum by Monteiro et al. (2000) yielded $\mathrm{He} / \mathrm{H} 0.035$ dex higher than assumed, O/H 0.2 dex lower and $\mathrm{N} / \mathrm{O} 0.1$ dex higher, but no $[\mathrm{O} \mathrm{II}]$ is given and the electron temperature is determined from [O III]. For the high excitation model A (of either geometry) by Morisset et al. (2000) the helium abundance could not be derived due to absence of the $\mathrm{He} \mathrm{I}$ line, $\mathrm{O} / \mathrm{H}$ was 0.1 dex lower and N/O 0.3 dex higher, but the [O II] 3728 lines are used. While this reflects the results of Gruenwald \& Viegas (1998), it does not seriously affect the classification of our object as type I.

\subsection{Comparison with other type I nebulae and photoionization modeling}

While the plasma diagnostics is an efficient means of interpretation, its results are dependent on weak diagnostic lines and (in some cases not very accurate) corrections for unseen stages of ionization. Additionally, it does not provide a check for a physically consistent description of either ionization or thermal balance in the nebula. In the following, we compare the spectrum with those computed with photoionization models and those observed in other type I nebulae. Here we do not intend to construct an individual detailed model for our object which can account for all the observational data. Because of the limited quality of our spectral data, we concentrate on the gross features. For this reason we shall restrict ourselves to rather simple models.

We use the code GWYN (details may be found in Köppen 1979; Rauch et al. 1996; Tajitsu et al. 1999) to compute spherically symmetric, dust-free nebulae in ionization and thermal equilibrium with the radiation from the central star. The nebula shell is assumed to be a filled sphere of constant density and chemically homogeneous. We shall neglect any density or temperature fluctuations, as well as any dynamical effects. Certainly, the complex, definitely non-spherical geometry of our nebula cannot be modeled by a one-dimensional code, and thus the comparison with the observed spectrum will be somewhat limited. But it may provide valuable first constraints for a future much more dedicated modeling.

In the absence of any direct information about the central star which would be essential to fix stellar parameters such as surface gravity and chemical composition, we represent the central star for simplicity by a blackbody spectrum of specified temperature and luminosity. In this context, the models serve as an interpolation device, not as a tool to derive e.g. the stellar temperature. The diffuse ionizing radiation due to emission by all relevant ions is treated in the outward-only approximation.

The He II 4686 line is stronger than $\mathrm{H} \beta$. Since in ionization bounded nebulae this line cannot be more intense than about 60 percent of $\mathrm{H} \beta$ (Stasińska \& Tylenda 1986), this is a clear indication that the nebula is strongly density bounded. It also means that it is impossible to derive the stellar temperature from this line ratio. Hence, we compute various grids of models, varying the temperature of the central star (between 80 and $200 \mathrm{kK}$ ) and its luminosity $\left(100,10^{3}\right.$ and $\left.10^{4} L_{\odot}\right)$. For the chemical composition of the nebula we use solar abundances: $\mathrm{He}=11.0$, $\mathrm{N}=8.07$ and $\mathrm{O}=8.83$ (in the usual logarithmic notation $12+\log \mathrm{X} / \mathrm{H})$. The other set, to represent a type I composition, has enhanced helium (11.2) and nitrogen (8.57) but depleted oxygen (8.33).

The data of the 19 other type I bipolar PNe (listed in Corradi \& Schwarz 1995) are compiled from: Aller \& Keyes (1987), Peimbert \& Torres-Peimbert (1987), Shaw \& Kaler (1989), Kingsburgh \& Barlow (1994), Perinotto et al. (1994), Costa et al. (1996), Corradi et al. (1997) and Perinotto \& Corradi (1998). Many of these nebulae have large angular sizes, and thus the spectroscopic observations pertain to only a small region. Depending on the position, the high or low ionization lines may thus be under- and overestimated. In the plots we have usually taken the data from central regions, thus e.g. He II will tend to be too strong and [O II] too weak. For NGC 2440 we show the data from all 6 regions observed by Perinotto \& Corradi (1998).

In Fig. 4 we present results on the He II 4686 and He I 5876 lines. The two grids of ionization bounded models show that the He II line intensity remains far below the value observed in our PN, while the HeI line is essentially sensitive to the helium abundance. For high temperature, low luminosity stars the models exhibit a slight enhancement of the HeI line, because in these ionization bounded models the $\mathrm{He}^{+}$zone extends somewhat further than the $\mathrm{H}^{+}$-Strömgren-sphere. As a contrast, all density bounded models of a given helium abundance are essentially confined to a straight line in this plot, because here both helium and hydrogen are ionized in the entire volume determined by the outer boundary, and the position of a nebula on the locus is determined by what fraction is filled with the $\mathrm{He}^{++}$zone. Since the recombination lines are not very sensitive to the electron temperature, details 


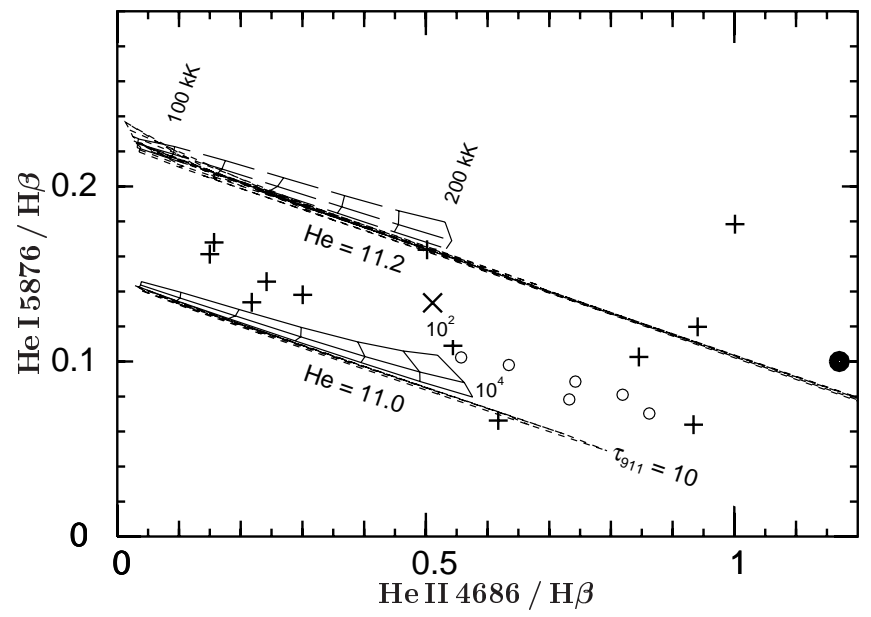

Fig. 4. The intensities of HeI 5876 and He II 4686 lines computed with spherical photoionization models of different stellar temperatures (from 80 to $200 \mathrm{kK}$ ) and luminosities (from 100 to $\left.10^{4} L_{\odot}\right)$ for nebulae with gas density $1200 \mathrm{~cm}^{-3}$ and different helium abundances. The full lines represent a grid of ionization bounded models of solar composition, dashed lines refer to ionization bounded models with type I composition. The dashed elongated lines are the corresponding grids for density bounded models. Also shown is the position of our PN (filled circle) in relation to PN G321.6-02.2 ("×", Corradi et al. 1997), several regions in NGC 2440 (small circles, Perinotto \& Corradi 1998), and other type I PNe ("+").

of the model do not matter much. Therefore, one obtains a simple expression for the straight line locus:

$\frac{\mathrm{He} \mathrm{I} 5876 / \mathrm{H} \beta}{1.45}+\frac{\mathrm{He} \mathrm{II} 4686 / \mathrm{H} \beta}{12.0}=\frac{n(\mathrm{He})}{n(\mathrm{H})}$

which depends on the helium abundance $n(\mathrm{He}) / n(\mathrm{H})$ (solar value 0.1 ). For our $\mathrm{PN}$ we get a helium abundance of 0.17 corresponding to a logarithmic value of 11.22 , as derived via the plasma diagnostics. Such a large value is found also in a few other objects: He 2-111, NGC 6537 and $\mathrm{Mz} 1$ all are beyond the locus for 11.2. For NGC 2440 we depict the various regions observed by Perinotto \& Corradi (1998); all show very much the same helium abundance, somewhat below 11.1.

In Fig. 5 we display for the same model grids and objects the intensity ratio of $[\mathrm{NII}] /[\mathrm{OII}]$ as a function of the helium abundance given by the simple relation Eq. (1). As $\mathrm{N}^{+}$and $\mathrm{O}^{+}$ions exist in the same zone, this line ratio measures quite directly the $\mathrm{N} / \mathrm{O}$ abundance ratio. This is clearly shown here. For our $\mathrm{PN}$ one needs a $\mathrm{N} / \mathrm{O}$ ratio about 3 times the solar value, $\log \mathrm{N} / \mathrm{O} \approx-0.25$. The plasma diagnostics gave a much higher value of +0.42 , because of the rather high electron temperature derived from the weak [N II] 5755 line whose intensity was most probably overestimated (see below). Though our PN appears to be somewhat more extreme than NGC 2440, PN G321.6-02.2 ("×", Corradi et al. 1997) requires an even higher $\mathrm{N} / \mathrm{O}$ ratio while $\mathrm{He} 2-111$ is more extreme in terms of the helium abundance.

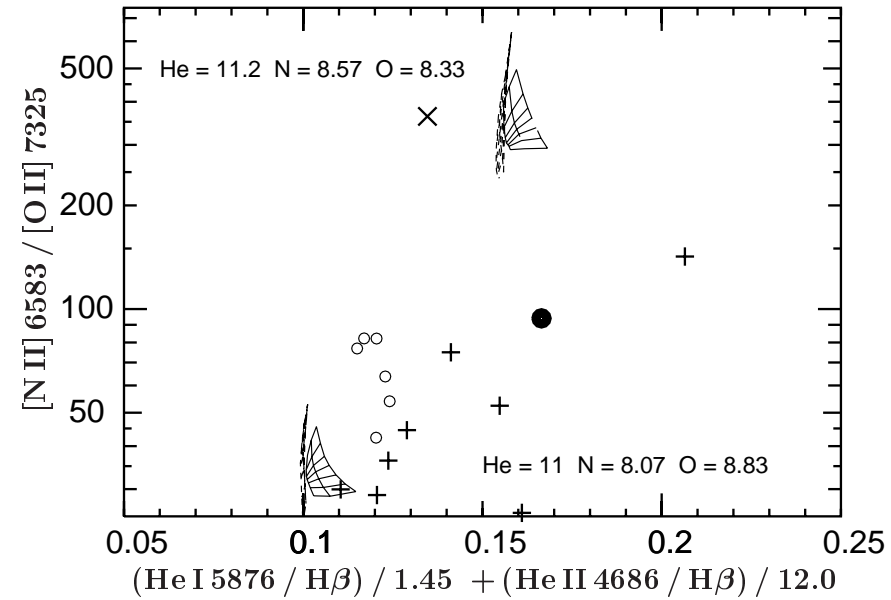

Fig. 5. Similar to Fig. 4, but for the line ratio [N II] 6583/ [O II] 7325 versus the combination of He II and He I line intensities which measures the He abundance.

The small spread of the models in this figure is caused by two effects: The derivation of helium abundances from the recombination lines by plasma diagnostics is quite reliable (and the quantity on the abscissa is a simplified form of it), provided there are no substantial pockets of neutral helium present, as would be the case in low excitation objects. This is also shown by Gruenwald \& Viegas (1998; their Fig. 4). The form of the stellar flux distribution influences the ratio of volumes of the $\mathrm{He}^{++}$and $\mathrm{He}^{+}$zones, but not the sum which is what determines the helium abundance. Secondly, $\mathrm{N}^{+}$and $\mathrm{O}^{+}$share almost identical ionization zones because of their close ionization potentials. Since this is determined by atomic physics, one would expect this to hold in more general situations other than spherical nebulae. The differences seen in the figure are due to the emissivity of [O II $] 7325$ depending more strongly on electron temperature than [N II] 6583. This is because the [O II] 7325 emission is governed by collisional excitation to the ${ }^{2} \mathrm{P}$ level which is high above ground level. The intensity ratio [N II] 6583/[O II] 7325 increases by about 30 percent when the electron temperature is lowered from $10000 \mathrm{~K}$ to $9000 \mathrm{~K}$.

Our PN has a very strong He II line but a rather weak [O III] line, which could be an indicator for a density bounded nebula. Figure 6 compares the computed line ratios $\mathrm{He} \mathrm{II} / \mathrm{He} \mathrm{I}$ and $[\mathrm{OIII}] /[\mathrm{OII}]$, which are insensitive to the abundances, with the observed values. Ionization bounded, solar abundance models are unsuitable to explain the observations, as the He II line cannot get sufficiently strong. Changing the helium, nitrogen and oxygen abundances to a type I PN pattern does not change the situation at all. Density bounded, solar abundance models give $\mathrm{HeII} / \mathrm{HeI}$ as large as needed to match our PN, but the $[\mathrm{O} \mathrm{III}] /[\mathrm{O} \mathrm{II}]$ ratio comes out far too large because of the cutting of the $\mathrm{O}^{+}$zone. This behaviour is typical for spherically symmetric models, and so this plot might be taken as a diagnostic for the nebular geometry. So far, little can be said whether this or a similar diagram would 


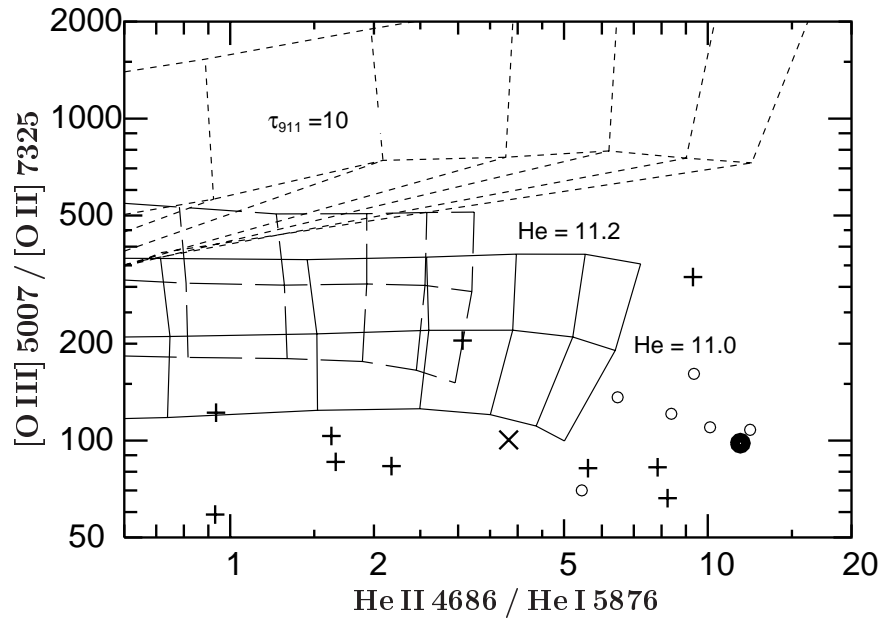

Fig. 6. Similar to Fig. 4, but for the line ratio [O III] 5007/ [O II] 7325 plotted against He II 4686/He I 5876.

allow definitive conclusions about the nebula geometry, in particular since ionization ratios depend also on the stellar flux distribution and how much the ionizing radiation field is modified by any internal dust. With respect to the other type I objects, our PN is quite extreme but the spectra from NGC 2440 have very similar line ratios.

The electron temperature sensitive line ratio $[\mathrm{NII}]$ $6583 / 5755$ is by about 30 percent lower than the average one observed in other nebulae as well as from models. Most likely the intensity of the weak $5755 \AA$ line has been overestimated. A temperature of $10000 \mathrm{~K}$ seems more appropriate, the derived chemical composition is presented in the third column of Table 3. The lower temperature increases mainly [O III] 5007, thus bringing up the oxygen abundance. The $\mathrm{N} / \mathrm{O}$ ratio becomes $\log \mathrm{N} / \mathrm{O}=+0.1$, more consistent with Fig. 5, but still a factor 2 larger, similar to the results of Gruenwald \& Viegas (1998).

The comparison with the sulphur lines shows that the estimates for the [S III] lines given in Sect. 4.1 would be quite acceptable both for models and other type I objects. Nonetheless, we do not accept them for any analysis.

Our PN has a $[\mathrm{Ar} \mathrm{V}] /[\mathrm{Ar} \mathrm{III}]$ line ratio as high as one would expect from the other nebulae for such a strong He II line.

All in all, the spectrum of our object does not show any serious deviations from other type I nebulae. The intensity of the [N II] 5755 line must have been overestimated, and thus the electron temperature used in the plasma diagnostics. Hence, the N/O ratio is most likely not as high as deduced from the plasma diagnostic, but it still is large enough to make our PN a genuine type I object in the definition of Peimbert \& Torres-Peimbert (1987).

\section{The nature of PN G291.4-00.3}

\subsection{Position in the galaxy}

One rough estimate of the distance is obtained by application of a simple Shklovski method: assuming a nebular

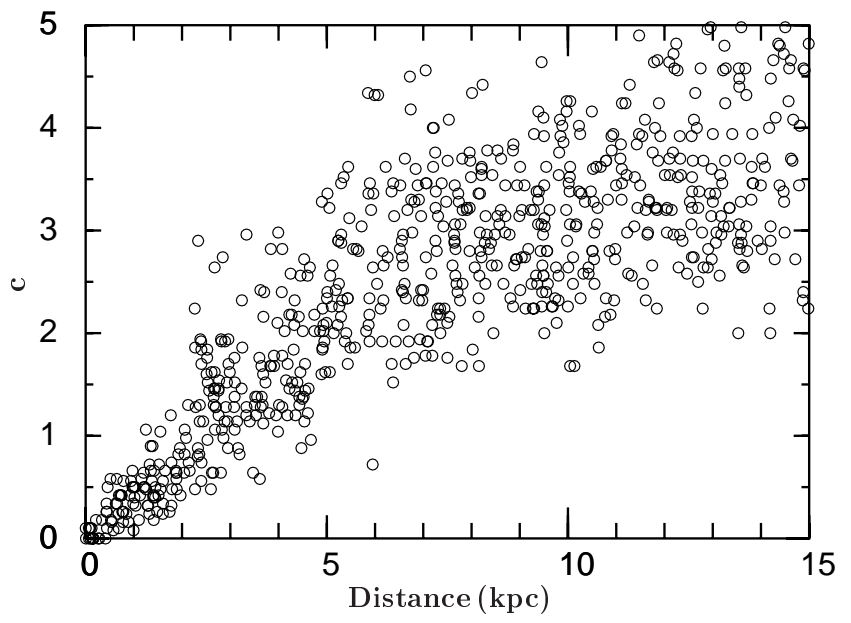

Fig. 7. The relation between extinction $c$ and distance of simulated objects in the direction of PN G291.4-00.3, expected from a model for the Galactic extinction by small clouds (Köppen \& Vergely 1998).

ionized mass of $0.2 M_{\odot}$ and a volume filling factor of 0.75 , one gets from the observable $\mathrm{H} \beta$ flux and with an angular diameter of $11^{\prime \prime}$ (which roughly covers the whole emission seen in Fig. 1) a distance of $3.2 \mathrm{kpc}$. However, as the distance decreases with the $5 / 3$ rd power of the angular diameter, the rather ill-defined size of the nebula suggests that this value may be rather uncertain.

An independent constraint can be derived from the measured extinction to the object: from simulations of observations in the direction of our PN with a statistical Galactic extinction model based on the extinction due to two populations of small clouds (Köppen \& Vergely 1998), one obtains a mean relation between extinction and distance as shown in Fig. 7. A value of $c \sim 3.8$ would be expected for objects in a rather large range, between about 5 and $15 \mathrm{kpc}$ away.

This model does not take into account any existing localized structures, such as spiral arms. The line-of-sight toward the PN crosses tangentially the Sagittarius-Carina spiral arm (Georgelin \& Georgelin 1970). The NGC 3603 giant H II region is located roughly at the more distant intersection on the same line-of-sight. A foreground color excess $E(B-V)$ of about 1.5 has recently been measured for NGC 3603 (Girardi et al. 1997). The PN's $E(B-V)$ of 2.6 hence suggests a lower limit of about $6-7 \mathrm{kpc}$ for the distance of PN G291.4-00.3.

Thirdly, the heliocentric radial velocity measured with the [N II] doublet lines in Cuisinier's high resolution spectra is $+5 \pm 1.5 \mathrm{~km} \mathrm{~s}^{-1}$. If we consider that the star follows closely the galactic differential rotation, then - in the simple case of a flat rotation curve with $R_{\odot}=8.5 \mathrm{kpc}$ and $v_{\odot}=220 \mathrm{~km} \mathrm{~s}^{-1}-$ this value gives two possible solutions for the heliocentric distance: $0.7 \mathrm{kpc}$ (rather unlikely given the large interstellar extinction provided by the nebular data) and $5.5 \mathrm{kpc}$. The second value is in good agreement with that obtained for the neighboring H II region 
NGC 3603 from radio observations $(d=6.1 \pm 0.6 \mathrm{kpc}$; De Pree et al. 1999).

All preceeding arguments show that the distance remains quite uncertain. For further analysis we shall rather adopt a distance of $6 \mathrm{kpc}$.

We can check the consistency of this distance with the other observational data: from the measured total $\mathrm{H} \beta$ flux $F(\mathrm{H} \beta)$, the interstellar extinction $c$, the distance $D$ and the density $n \approx n_{\mathrm{p}} \approx n_{\mathrm{e}}$, one gets the emitting volume $V$ from

$4 \pi D^{2} \cdot F(\mathrm{H} \beta) \cdot 10^{c}=h \nu(\mathrm{H} \beta) \cdot n_{\mathrm{p}} n_{\mathrm{e}} \cdot \alpha_{\mathrm{eff}}\left(T_{\mathrm{e}}\right) \cdot \epsilon V$

with the effective recombination coefficient $\alpha_{\text {eff }}\left(T_{\mathrm{e}}\right)$ for the $\mathrm{H} \beta$ line (cf. Osterbrock 1974), and the filling factor $\epsilon$ denoting what fraction of the full sphere volume $(V=$ $4 \pi R_{\text {neb }}^{3} / 3$ ) is emitting. One may thus compute the angular diameter of the region in $\operatorname{arcsec} d=2 \cdot 206265 R_{\text {neb }} / D$, where $R_{\text {neb }}$ is given in parsec. To evaluate this and other derived formulae and their inverse relations, and to obtain other parameters as well, an interactive Java applet is available on the $\mathrm{Web}^{2}$. With the values for our PN and a unity filling factor, we get an angular diameter of $14^{\prime \prime} 6$ for a nebula density of $1200 \mathrm{~cm}^{-3}$ or $11^{\prime \prime}$ for $1800 \mathrm{~cm}^{-3}$. If we had used a distance of $3 \mathrm{kpc}$, the density would be $2500 \mathrm{~cm}^{-3}$. Most likely, this slight inconsistency is caused by the $\mathrm{H} \alpha$ flux having been overestimated, as it was extrapolated from the area covered by the slit to the whole nebula. Another possibility is that due to the overall outward decrease of density, the mean density in the $\mathrm{H}^{+}$zone is somewhat larger than what is measured by the [S II] line ratio. Thus we believe that the values for distance, angular size and density are resonably consistent with each other.

It is worth noting that in this estimate we could use a unity volume filling factor, while the complex shape of the nebula would suggest a smaller value. Checks with other type I nebulae gave inconclusive results, as the total flux of $\mathrm{H} \beta$ emission from the large nebulae with complex shapes is also not measured directly.

\subsection{Evolutionary status of nebula and central star}

Since the nebula is certainly density bounded, no direct information about the central star's temperature can be drawn from e.g. the strength of the He II 4686 line. However, given the adopted distance, we may use the observed flux in the He II line as a constraint on temperature and luminosity of the central star. Following Harman \& Seaton (1966) the number of photons emitted per unit time by the nebula in this recombination line

$$
\begin{aligned}
\frac{4 \pi D^{2} \cdot F_{0}(\mathrm{He} \text { II 4686) }}{h \nu(\mathrm{He} \text { II 4686) }} & =n\left(\mathrm{He}^{+}\right) \cdot n_{\mathrm{e}} \cdot \alpha_{\mathrm{eff}}\left(T_{\mathrm{e}}\right) \cdot V \\
& =\mathcal{N}\left(\mathrm{He}^{+}\right) \cdot \alpha_{\mathrm{eff}}\left(T_{\mathrm{e}}\right) / \alpha_{\mathrm{A}}\left(T_{\mathrm{e}}\right)
\end{aligned}
$$

\footnotetext{
2 http://astro.u-strasbg.fr/ Noppen/stroemgren/ Stroemgren.html
}

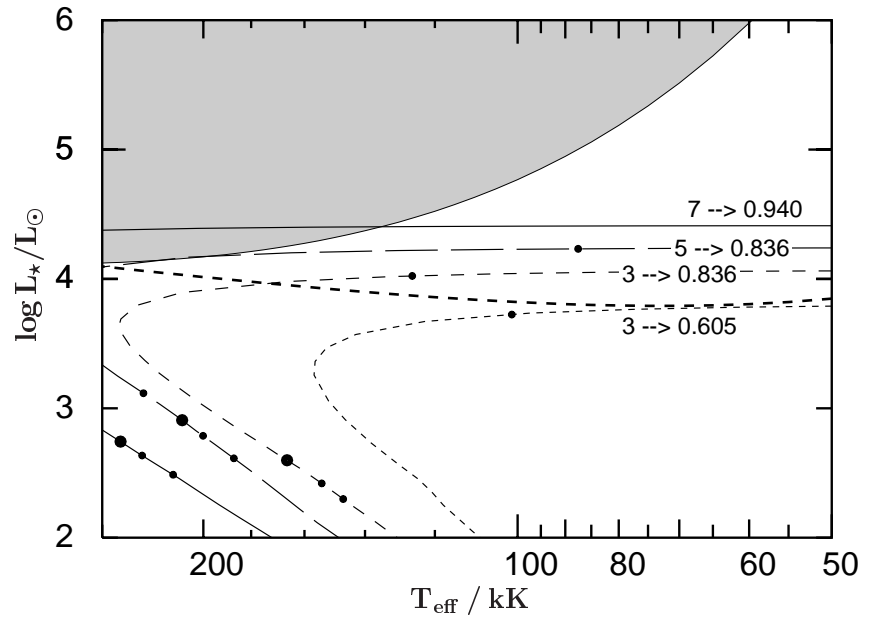

Fig. 8. Constraints for the position of the central star of PN G291.4-00.3 in the HR diagram, obtained from the observed flux of the He II 4686 line (shaded area) and the H $\alpha$ flux (thick dashed line). A distance of $6 \mathrm{kpc}$ is assumed. We also depict evolutionary tracks of PN central stars (Blöcker 1995): filled circles indicate the ages of 200, 500, 1000 (large), 2000 and 5000 years after leaving the AGB. The tracks are identified with their initial and final masses.

is proportional to the number of photons emitted by the central star per unit time which are capable of ionizing $\mathrm{He}^{+}$:

$\mathcal{N}\left(\mathrm{He}^{+}\right)=4 \pi R_{*}^{2} \cdot \int_{54 \mathrm{eV}}^{\infty} \frac{F_{\nu}^{*}(\nu)}{h \nu} \mathrm{d} \nu$

Equation (4) applies strictly for nebulae which are optically thick in the He II Lyman continuum and that the nebula absorbs all photons produced by the star. In our density bounded object the fraction of ionizing photons which do not lead to a recombination line photon may well be substantial, and thus the central star's luminosity could be higher. Likewise, any substantial absorption of ionizing photons by dust in the nebula would push up the required luminosity by that amount.

If we assume for simplicity blackbody spectra, we obtain a constraint for the position of the central star in the HR diagram, shown as the shaded area in Fig. 8. Applying the same argument to the flux of the hydrogen recombination lines, one gets as a lower limit for the luminosity the thick dashed curve, which does not give any further constraint. Comparison with theoretical evolutionary tracks of post-AGB stars shows that our PN's central star would be compatible with being on a track of a massive progenitor star. While it seems tempting to associate the central star with the most massive track, one has to keep in mind the rather uncertain distance, the limited quality of the available data and the unknown fraction of absorbed photons. If e.g. the object was as close as $3 \mathrm{kpc}$, the constraint region would be placed towards luminosities four times lower, thus giving a much greater overlap with the tracks. Moreover, changes of the same size are also to be expected from the unknown filling factor, dust absorption 
and deviations of the true flux distribution of the central star from that of a blackbody.

Therefore, we shall take this constraint as a confirmation that the central star is a genuine post-AGB object, but not yet as a good measure of its mass.

\section{Conclusion}

Optical imaging and spectroscopy confirm that the emission line object found on WFI images is a genuine planetary nebula (PN G291.4-00.3), and that it is a highexcitation bipolar type I nebula. The chemical composition shows enhanced helium and nitrogen. Oxygen, sulphur and argon abundances seem to be solar.

The radial velocity derived from the [N II] lines indicates that the PN might be associated with the giant $\mathrm{H}$ II region NGC 3603.

From the He II luminosity, some constraints on the central star can be placed: it is rather hot (at least $100 \mathrm{kK}$ ) and luminous (at least about $3000 L_{\odot}$ ). This is quite consistent with its origin from a fairly massive main sequence star (about $5 M_{\odot}$ ). Such a star would have passed through the phase of thermal pulses on the AGB, and thus would have synthesized and ejected nitrogen as well as helium.

Acknowledgements. We thank the ESO staff for support during the observations, François Cuisinier for placing at our disposal his observations, Agnès Acker and Michael Bremer for help during data reduction and many fruitful discussions, and Jim Caswell for cross-checking the PN position with his catalogues of maser sources. We appreciate the comments and suggestions provided by our referee Stefan Kimeswenger, which led to a substantial improvement of the manuscript. This research has been supported by the Deutsche Forschungsgemeinschaft (DFG) in the framework of its "Physics of Star Formation" program under grants Yo 5/23-1, Zi 242/9-1 and Zi 242/22-2. SD acknowledges a grant from FAPESP. DN also acknowledges support by IRAM, Grenoble.

\section{References}

Acker, A., Köppen, J., Stenholm, B., \& Jasniewicz, G. 1989, A\&AS, 80, 201

Acker, A., Ochsenbein, F., Stenholm, B., et al. 1992, Strasbourg-ESO catalogue of Galactic Planetary Nebulae, ESO
Aller, L. H., \& Keyes, C. D. 1987, ApJS, 65, 405

Balick, B. 1987, AJ, 94, 671

Beichman, C. A., Neugebauer, G., Habing, H. J., Clegg, P. E., \& Chester, T. J. 1988, Infrared astronomical satellite (IRAS) catalogs and Atlases

Blöcker, T. 1995, A\&A, 299, 755

Caswell, J. L. 1998, MNRAS, 297, 215

Ciardullo, R., Bond, H. E., Sipior, M. S., et al. 1999, AJ, 118, 488

Corradi, R. L. M., \& Schwarz, H. E. 1995, A\&A, 293, 871

Corradi, R. L. M., Villaver, E., Mampaso, A., \& Perinotto, M. 1997, A\&A, 324, 276

Costa, R. D. D., Chiappini, C., Maciel, W. J., \& de Freitas Pacheco, J. A. 1996, A\&AS, 116, 249

Cuisinier, F., Acker, A., \& Köppen J. 1996, A\&A, 307, 215

De Pree, C. G., Nysewander, M. C., \& Goss, W. M. 1999, AJ, 117,2902

Dopita, M. A., \& Meatheringham, S. J. 1990, ApJ, 357, 140

Georgelin, Y. P., \& Georgelin, Y. M. 1970, A\&A, 7, 133

Girardi, L., Bica, E., Pastoriza, M. G., \& Winge, C. 1997, ApJ, 486,847

Gruenwald, R., \& Viegas, S. M. 1998, ApJ, 501, 221

Harman, R. J., \& Seaton, M. J. 1966, MNRAS, 132, 15

Hog, E., Fabricius, C., Makarov, V. V., et al. 2000, A\&A, 355, L27

Kennicutt, R. C. 1984, ApJ, 287, 116

Kingsburgh, R. L., \& Barlow, M. J. 1994, MNRAS, 271, 257

Köppen, J. 1979, A\&AS, 35, 111

Köppen, J., \& Vergely, J.-L. 1998, MNRAS, 299, 567

Köppen, J., Acker, A., \& Stenholm, B. 1991, A\&A, 248, 197

Kraan-Korteweg, R. C. 2000, A\&AS, 141, 123

Monteiro, H., Morisset, C., Gruenwald, R., \& Viegas, S. M. 2000, ApJ, 537, 853

Morisset, C., Gruenwald, R., \& Viegas, S. M. 2000, ApJ, 533, 931

Osterbrock, D. 1974, Astrophysics of Gaseous Nebulae (W. H. Freeman, San Francisco)

Peimbert, M., \& Torres-Peimbert, S. 1987, Rev. Mex. A\&A., 14,540

Perinotto, M., \& Corradi, R. L. M. 1998, A\&A, 332, 721

Perinotto, M., Purgathofer, A., Pasquali, A., \& Patriarchi, P. 1994, A\&AS, 107, 481

Rauch, T., Köppen, J., \& Werner, K. 1996, A\&A, 310, 613

Shaw, R. A., \& Kaler, J. B. 1989, ApJS, 69, 495

Stasińska, G., \& Tylenda, R. 1986, A\&AS, 155, 137

Tajitsu, A., Tamura, S., Yadoumaru, Y., Weinberger, R., \& Köppen, J. 1999, PASP, 111, 1157 